\title{
Mística, ciencia y política en la construcción de sistemas de salud. La experiencia de Chile
}

\author{
Jorge Jiménez de la Jara, MSP. ${ }^{(1)}$
}

\begin{abstract}
Jiménez-de la Jara J.
Mística, ciencia y política

en la construcción de sistemas de salud.

La experiencia de Chile.

Salud Publica Mex 2001:43:485-493.

El texto completo en inglés de este artículo está disponible en: http://www.insp.mx/salud/index.html
\end{abstract}

\section{Resumen}

Partiendo de la premisa de que la conjunción de mística, ciencia y política -escalones para un bien común destinado a la humanidad- potencia la resolución de objetivos socialmente planteados, el presente documento revisa una parte medular de la experiencia en el Sector Salud en C hile, cuyos procesos hacia la primera mitad del siglo XX, como el nacimiento del Servicio $N$ acional de Salud y el desarrollo de políticas de atención materno infantil, figuran como parteaguas del actual sistema de salud chileno. Luego de la catastrofe ocasionada por el sismo de 1939, el futuro presidente de la nación, doctor Salvador Allende, establece el Consejo Especial de Salubridad, que equilibra políticas e impulsa una reforma a la Seguridad Social que enfrentó obstáculos de financiamiento y el resquemor de los médicos opuestos a la socialización de la práctica médica. En 1951 se aprueba dicha reforma la cual amplía la cober tura a to dos los obreros y a sus familias, separando la previsión de la seguridad en salud, enfatizando en la atención pediátrica preventiva, en la materno infantil y en salud reproductiva. Esta reforma postuló el derecho a la salud, la solidaridad y la equidad como principios sustanciales en cualquier formulación político-programática de salud. El autor se pregunta si los desarrollos posteriores de la seguridad social en salud en su país han sido consecuentes con estos principios. El texto completo en inglés de este artículo esta disponible en: http://www.insp.mx/salud/index.html

Palabras clave: sistemas nacionales de salud; mística; ciencia; política; Chile

\author{
Jiménez-de la Jara J. \\ Inspiration, science, and politics \\ in the creation of a health system: \\ The Chilean experienc. \\ Salud Publica Mex 2001:43:485-493. \\ The English version of this paper \\ is available at: http://www.insp.mx/salud/index.html
}

\section{A bstract}

The combination of inspiration, science, and politics is a cornerstone precept for the common good of humanity, towards the fulfillment of social objectives. Based on this precept, this paper reviews core experiences of the C hilean $\mathrm{H}$ ealth Sector. H ealth sector key events taking place during the first half of the 20th century were the creation of the $\mathrm{N}$ ational Health Service and the development of mother and child healthcare policies. After the earthquake of 1939, the future President of Chile, D octor Salvador Allende, set up the Special Sanitation Council, to balance policies.Also, he launched the Social Security reform process, which endured financing restrictions and the animosity of physicians opposing the so cialization of medical care. In 1951 the reform was approved, to extend coverage to blue collar workers and their families; separate health provision from healthcare security; emphasize preventive pediatric care in mother's health, and reproductive health. The basic tenets of healthcare reform were the right to health, solidarity, and equity, as the pillars of policy-making and healthcare programming. The question of whether the evolution of social security in Chile has been consistent with the original healthcare reform tenets is raised by the author. The English version of this paper is available at: http:// www.insp.mx/salud/index.html

Key words: national health systems; mystic; science; politics; Chile

Trabajo presentado en la Conferencia José Luis Bobadilla, IX Congreso Mexicano de Salud Pública, Cuernavaca, Morelos, México, 6 de marzo de 2001.

(1) Departamento de Salud Pública, Universidad Católica de Chile. Presidente del Consejo Ejecutivo de la O rganización Mundial de la Salud.

Solicitud de sobretiros: MSP. Jorge Jiménez de la Jara. D epartamento de Salud Pública, Universidad Católica de Chile.

Marcoleta 352, Santiago de Chile.

Correo electrónico: jjimenez@ med.puc.cl 
E ste trabajo corresponde a la Conferencia Magistral José Luis Bobadilla dictada el 6 de marzo de 2001, durante el IX Congreso Nacional de Investigación en Salud Pública, efectuado en el Instituto Nacional de Salud Pública, Cuernavaca, Morelos, México. Por considerarlo de interés para sus lectores, Salud Pública de México lo reproduce en su formato original.

Para mí es un gran honor estar aquí con ustedes recordando a José Luis Bobadilla con una conferencia en su memoria.

José Luis fue un hombre muy especial en un tiempo muy particular de la salud pública en México y en el mundo. Pocos hombres tienen la ocasión de ser grandes en una época de oportunidades y desafíos trascendentales como la que a nuestro amigo le tocó en su paso material por este mundo.

Digo su paso material porque ciertamente es el espíritu el que permanece fuerte, sólido y José Luis estaba dotado principalmente de espíritu. Un espíritu que trasuntaba nítidamente desde el primer contacto que él iluminaba con una mirada que era mezcla de ingenuidad y modestia con certidumbre y fuerza intelectual. Rara mezcla en verdad pues la sabiduría se asocia más frecuentemente con la soberbia y la arrogancia.

Más allá de su inteligencia y su capacidad de trabajo, el Bobadilla que yo conocí fue un joven brillante especialmente apto para las relaciones interpersonales, un hombre acogedor, transparente y amistoso, con el cual era muy fácil establecer relación. Generoso en el elogio de los demás y sus virtudes, parco y modesto en la apreciación de sí mismo.

La presencia de José Luis Bobadilla en la salud pública mexicana e internacional por otra parte, es una función directa de su imagen encarnada en una generación de élite de la cual muchos miembros distinguidos están aquí presentes, precisamente en el auditorio que lleva el nombre del Padre Fundador de esta pléyade de intelectuales y políticos de la salud pública, el maestro Guillermo Soberón.

Esta gran riqueza humana de José Luis me hacía atribuirle un gran futuro en la vida pública, porque su sentido místico de la salud pública era evidente y la mística es central en las empresas humanas. Mística tomada no tanto en su relación con la divinidad, sino más bien con la búsqueda de un bien superior. Mística que, como condición del espíritu se constituye en la fuerza y el motor de las ideas. Mística que en la definición de la Real Academia es aquello que incluye algún misterio o razón oculta que trasciende a la razón habitual. Es ciertamente la trascendencia de la fuerza interior.
Hoy José Luis es parte del conjunto de los héroes de las mil caras, como dice Campbell. ${ }^{1}$ De aquellos héroes que inspiran y orientan a los jóvenes en la persecución de los grandes objetivos de la humanidad. Y que en esta persecución necesariamente enfrentan el sacrificio y el martirio como capítulo final de su presencia terrena, transportándose así al lugar superior de las figuras inspiradoras que permanecen en el tiempo.

Los héroes encarnan un mito que se nutre místicamente y alimenta de la leyenda que le circunda. El material del mito, según el mismo Campbell, "es el material de nuestra vida, el material de nuestro cuerpo y el material de nuestro ambiente, y una mitología viviente y vital, se relaciona con ellos en términos apropiados para la naturaleza del conocimiento de su tiempo". ${ }^{2}$ Y prosigue: "en la primordial relación entre un hijo y su madre, está la imagen básica de la mitología, aquella que Le Debleu llamó la participación mistica, participación mística entre la madre y el niño y el niño y su madre, donde está el último territorio de la felicidad". El antropólogo prosigue después elaborando que la tierra y el universo son para el adulto el terreno de su experiencia mítica y apunta que "las sociedades que no tienen un mito que les sostenga y dé coherencia, entran en proceso de disolución", para insistir que hay notorias diferencias entre mito y mística comparados con una ideología y su ejecución pragmática.

\section{Grandes objetivos de la humanidad}

La vida y la salud son parte de los grandes objetivos del hombre y de la humanidad. Vencer a la muerte, especialmente la muerte prematura e innecesaria, la muerte injusta. Y que las vidas sean llenas de salud, sin dolor, alegres y productivas.

Dar más años a la vida fue fórmula poética, bella y expresiva, según expresara la Organización Mundial de la Salud -OMS Europa- en su documento de Salud para Todos, de fines de los años setenta, como una forma trascendente de política para disminuir las muertes evitables. ${ }^{3}$

Y en esta misma línea se dijo Dar más Vida a los Años como una manera de expresar que la salud y la capacidad plena son parte importante de esa existencia más prolongada. Controlar y hacer más tolerable el dolor innecesario e injusto, desarrollar las potencialidades físicas, mentales y sociales de cada uno, aisladamente y en comunidad.

La mística religiosa tiene un equivalente en la mística social, aquella de los líderes del cambio por el bien 
de la humanidad. Esos hombres que tienen convicciones fuertes y duraderas, que les otorgan energías para batallar incansablemente por sus objetivos principales.

El derecho a la salud, solidaridad, equidad, son algunos de los principios frecuentemente enunciados en cualquier formulación político-programática de salud. Sin embargo, observamos que este enunciado pasa a ser a menudo un ritual que, desgraciadamente, tiende a perder fuerza en sus expresiones concretas.

Los principios y las doctrinas viven en las personas y en sus conductas, mucho más que en el papel o en las declaraciones. Es el compromiso personal, la trayectoria y el esfuerzo de cada uno de nosotros lo que cuenta para poder desarrollar procesos que nos lleven al cumplimiento de los objetivos. Sin embargo, las expresiones de la mística en la acción social y política, también se expresan en declaraciones líricas, a menudo poéticas, que encabezan los programas y discursos de reforma social. Cuanto de estas declaraciones sea creíble, será materia del líder y su público.

A la mística, le siguen la ciencia y su método, la información y el conocimiento de los hechos y sus relaciones para buscar soluciones basadas en la correcta observación de cómo se hacen mejor las cosas. Es la evidencia para la política, como le bautizara el ministro Julio Frenk en su paso por la OMS.

A la ciencia, le sigue la política, cuyo principal medio es ser el arte del manejo del poder para un fin, ojalá superior y noble. En la política, el poder viene de la capacidad de manejo que se genera en el conocimiento del ser humano, de las relaciones interpersonales, del saber observar y escuchar, del sentido de la oportunidad y de la persistencia en las empresas. No se excluyen por cierto los aspectos empíricos de la política como ciencia y como aplicaciones de constantes y de métodos de búsqueda de información para la acción.

La política, sin embargo, es una actividad que a menudo es tomada por asalto para causas innobles, para las motivaciones egoístas o el simple abuso del poder. Quienes tienen grandes objetivos y causas nobles deben cuidarse de estos peligros.

La secuencia mística-ciencia-política debe expresarse en realidades específicas de una manera concreta, muchas veces compleja y difícil, en la construcción de un sistema de salud. Construcción que siempre se basará en los elementos del edificio anterior y agregará ladrillos nuevos, quizá mejor probados y menos costosos, para resolver un conjunto de problemas o un asunto particular.
Es sobre esto precisamente, el cómo se encarnan los principios y los métodos en personas y grupos concretos para obtener objetivos, de lo que deseo hablarles hoy.

Quiero contarles, un poco desde la historia cercana y un poco desde la experiencia personal, cómo veo la construcción de los sistemas de salud en un esfuerzo concertado de personas y de grupos.

\section{¿Cómo nace una idea o un proyecto de sistema de salud?}

Para ilustrar este punto tomaré dos procesos que ocurrieron en Chile, durante la segunda mitad del siglo $X X$, que son los que conozco mejor.

Uno es la concepción, nacimiento y desarrollo del Servicio Nacional de Salud, entre 1940 y 1980. El otro es el desarrollo de las políticas de atención maternal e infantil, las cuales ocurren en paralelo a la estructura sanitaria anterior.

En 1939 un terremoto, de aquellos fuertes y devastadores que ustedes y nosotros conocemos, asoló el centro-sur de Chile. Más de 30000 muertos y ocho provincias en el suelo fueron el balance de este sismo, que por cierto no fue ni el primero ni el último.

Como todos los terremotos y las grandes catástrofes naturales, el de 1939 provocó en Chile la agudización de un debate que ya venía sobre las desigualdades sociales y la pobreza, expresadas entre otras cosas, en el dramático diferencial de salud entre pobres y ricos, entre urbanos y rurales, entre educados y analfabetos. Nada nuevo, diría un observador.

Sí, salvo el drama y la energía social que aportan esos 30000 muertos y el desamparo de la miseria que, entre otros efectos, provocan la necesidad de buscar soluciones eficaces para enfrentar el dolor y el sufrimiento.

A la época existían en Chile tres entes preocupados de dar salud:

- Los servicios sanitarios del Estado, dispersos, centralistas, pobres de solemnidad aunque bien fundados en sus ideas, como los destinados a la Prevención.

- El Servicio del Seguro Obrero Obligatorio, nacido a la inspiración de Bismark en 1924, protector sólo de los trabajadores activos, con contrato y pago efectivo, eficiente pero excluyente en las tareas de la seguridad social, incluyendo salud.

- La Junta de Beneficiencia Pública, vieja organización de caridad privada, propietaria de los hospi- 
tales y algunos dispensarios, por aquella época receptora de subsidios crecientes por parte del Estado, pero con gestión privada y autónoma.

Ante el desastre, las autoridades optaron por disponer la unidad de mando operacional de todas las estructuras de salud, en las provincias afectadas por el sismo. Todas las estructuras, con un criterio militar, fueron centralizadas en una sola autoridad y se les llamó primero Servicios de Salubridad de Emergencia y después Servicios de Salubridad Fusionados.

El Comando Unico de los Servicios de Salud Fusionados para las provincias afectadas tenía su sede en Chillán, mientras que en la capital se organizó un Consejo Especial de Salubridad presidido por el Ministro, con representación de los servicios integrados. Este comando único ejercía sus tareas en el lineamiento de las políticas acordadas en conjunto por las autoridades a nivel central y en las zonas, con sus equipos técnicos cuya tarea era la de normalizar y estandarizar el trabajo de los servicios y contribuir al perfeccionamiento de los profesionales. ${ }^{4}$

Al evaluar la acción de estos Servicios de Salubridad Fusionados, se leía en su informe que los funcionarios habían:

inspeccionado 11173 viviendas de las cuales 9235 estaban en mal estado, sin servicios higiénicos o con pozos negros no funcionantes. Menos de la mitad de las casas tenía agua potable. Se desinfectaron las casas, se bañaron 26648 personas y se vacunaron contra la viruela a 14 000. Las epidemias quedaron bloqueadas en medio de los escombros y en materia de prestaciones, enfatizando el punto de la productividad, mostraban un cuadro en que las atenciones médicas subían de 41 mil a 77 mil, las recetas médicas despachadas de 47 mil a 91 mil y las curaciones de 64 mil a 131 mil.

El Ministro de Salud de la época y futuro presidente, doctor Salvador Allende, hacía notar que esta nueva "medicina social real", que atendía a todos sin distinción y con los mismos elementos científicos, le permitía afirmar que: "los hechos permiten afirmar, sin temor a ser rebatido, que la experiencia de fusión de servicios médicos asistenciales y sanitarios de la zona afectada por el terremoto, pese a las circunstancias extraordinariamente difíciles en que han comenzado y a los defectos e organización que han tenido, ha sido una empresa coronada por el éxito." ${ }^{5}$

Vemos así, que de la mística que provoca una tragedia como el terremoto, nace una medida que es observada y evaluada con los métodos de la época, tan válidos entonces como ahora, simples acumulaciones de datos estadísticos que generan una evaluación positiva de la medida de reorganización de los servicios de salud. Medida la cual, posteriormente, se intenta llevar a una aplicación nacional.

El pensamiento político era que el estado liberal asistencial había fracasado y que el estado social, de carácter orgánico e intervencionista, debía proseguir para enmendar los problemas de las clases desposeídas.

El mismo Salvador Allende tomó la iniciativa de reformar la seguridad social, con la creación de una comisión amplia en lo técnico y pluralista en lo político, para reformar la ley 4054, que había creado la Caja del Seguro Obrero Obligatorio, cuyos servicios médicos eran la parte central del sistema de salud chileno de entonces. La idea global del proyecto era la de dar "seguridad vital" a los trabajadores y sus familias frente a las incertidumbres del sistema liberal capitalista, mediante la extensión de los servicios médicos a las familias de los asegurados, pensiones vitales para invalidez y vejez, seguros de orfandad, viudez y cesantía, cambiado el sistema de capitalización por uno de reparto.

Este proyecto de reforma fue enviado al Congreso, sin hablar inicialmente de un Servicio Unico de Salud, a pesar de las evidencias antes anotadas. El proyecto pasó casi 10 años entre comisiones y subcomisiones del Congreso de Chile. No había madurez política, no había claridad sobre cómo financiar una nueva seguridad social. Tanto patrones como obreros no querían aportar más a los nuevos esquemas. La política y la politiquería tuvieron un largo periodo de influencia sobre esta reforma, bloqueándola con diversos mecanismos y argumentos. El financiero era uno de los principales. La deuda del Fisco con las cajas de previsión era de 40 millones de pesos, mientras que la Caja del Seguro Obrero con la Beneficencia, que administraba los hospitales, era de más de 20 millones de pesos de la época, en 1941. Comunistas culpaban a socialistas, radicales también culpaban a los socialistas de esta mala gestión. Duras campañas de prensa se realizaban en los medios nacionales. Una nueva administración inició la venta de activos prescindibles y de documentos financieros para cancelar deudas.

Nos podemos preguntar: ¿hay algo de nuevo en una historia tan vieja?

\section{Los médicos y la reforma}

Los médicos de la época, organizados en una poderosa asociación vieron cómo una eventual socialización de la medicina les amenazaba con reducir sus ingresos y públicamente decían: 
Empleados y obreros creen que el médico disfruta y aprovecha de un régimen que ellos tienen por derecho propio, olvidándose de que antes de que aceptáramos el sacrificio de nuestra profesión liberal para funcionalizarnos, ellos pagaban costosamente su situación de enfermos o imploraban una caridad vergonzante para obtener salud y vida.

No cabe a nuestro juicio otro camino que colocarnos también egoístamente en un lugar clasista y defender nuestros fueros, aun a trueque de que se pierda un caro progreso y un ideal que terminará por matarnos de hambre y amarguras. Si los poderes públicos, si las autoridades médicas, si los consejeros profanos y los propios beneficiados con nuestra obra no quieren aceptar que los médicos son hombres dignos, seleccionados por el estudio y trabajadores con derecho a la vida, lancemos nuestro reto y empuñemos nuestras armas para defendernos. ${ }^{6}$

Poco después, en 1948, se aprobaba la ley que daba estatuto de corporación de derecho público al gremio, mediante la creación del Colegio Médico, hasta la fecha buque insignia del corporativismo médico. Pero, esta concesión no era suficiente para el Colegio Médico, el cual negoció exitosamente un Estatuto del Médico Funcionario, mediante el cual se aseguraba a los profesionales, por ley de la República, remuneraciones excelentes para la época a cambio de su dedicación exclusiva a las tareas de la medicina estatal. Jocosamente se le llamó el "Estatuto del Médico Millonario".

\section{Creación del servicio nacional de salud}

En 1950, casi 10 años después de su presentación inicial, se retomó la discusión de las reformas a la seguridad social, las cuales incluían un importantísimo, aunque no valorizado, principio de separación de los fondos previsionales generales de los fondos para salud. Creo personalmente que ahí está uno de los detalles más importantes del éxito de la medicina social chilena hasta la fecha.

Curiosamente, la creación del Sistema Nacional de Salud (SNS) iba un poco de contrabando en la reforma previsional y así no tenía demasiado apoyo, pero tampoco tuvo finalmente oposición.

El nacimiento del SNS fue por aprobación unánime, bajo la diestra conducción de un cientista básico, el Profesor de farmacología Jorge Mardones Restat, Ministro de Salud social-cristiano. Sus testimonios, los cuales tuve el privilegio de recibir personalmente eran extraordinarios, pues este hombre aplicaba el método científico a las negociaciones políticas con los partidos y los gremios, pudiendo hacer complica- dos cálculos de costo y beneficio con viejas calculadoras tipo ábaco, que le permitían tener respuestas tan rápidas como paralizantes con sus interlocutores.

Finalmente, en 1951, cuando el proyecto de reforma fue aprobado por voto unánime en ambas cámaras, todos los partidos tomaron crédito de este consenso histórico. Lógico, ya que después de la Segunda Guerra Mundial, todos los países, encabezados por los triunfadores, quisieron hacer justicia social y equidad, aumentando las estructuras y la fuerza del Estado Benefactor y nuestros políticos no querían estar ajenos a esa tendencia mundial; además, dicho sea de paso y como una verdad permanente, 1951 era un año de elecciones presidenciales.

Pero más allá del oportunismo de estar a la moda o de lograr votos, había todo un largo proceso de maduración que permitía que una idea, después de una década encontrara el apoyo unánime

En la práctica, se amplió la seguridad social a los obreros y sus familias, mejorando sustancialmente los beneficios de corte tradicional frente a los riesgos clásicos de la vida y la enfermedad.

Se separó la previsión social de la previsión de salud, hecho al cual otorgo la máxima importancia, pues permitió integrar los servicios más desarrollados del Seguro Obrero, con aquellos más pobres de la Sanidad y la Beneficencia. Pensemos en tantos países en que la sobreposición de ambos fondos no permite claridad en las cuentas ni el fin de privilegios de algunos grupos.

El nuevo Servicio Nacional de Salud creaba una estructura unitaria de 16 servicios dispersos, e iniciaba una larga carrera de construirse a sí mismo a partir de esos componentes. Cada uno con su historia, sus mañas, sus prebendas, sus vicios y sus virtudes. La marcha que se iniciaba era necesariamente larga y agotadora, llena de obstáculos y dificultades. Producto de las inevitables transacciones con las fuerzas de la derecha, quedaron excluidos los servicios de accidentes laborales y no hizo extensivo el servicio de salud a los empleados de cuello y corbata. Ambos problemas quedarían pendientes para otros ciclos de reformas posteriores.

\section{La puesta en marcha del Servicio Nacional de Salud y sus primeros impactos}

El siguiente gobierno, de un signo populista y nacionalista, totalmente distinto al del precedente, una alianza de socialdemócratas con derechistas de sensibilidad social, tuvo que hacerse cargo de implementar esta nueva estructura de integración vertical perfecta entre el financiamiento y la provisión de servicios, el 
paradigma de la época. Pero además, una organización que pretendía aplicar los principios de la integralidad de la medicina, desde la prevención hasta la rehabilitación, principios antiguos pero siempre necesarios.

El grupo de especialistas que dio forma a la nueva estructura, era una generación de especialistas en salud pública, formados esencialmente mediante el proyecto de la Fundación Rockefeller que dio origen a las escuelas clásicas de salud pública, Harvard, Hopkins y Columbia, y a otras en el mundo, como la de la Universidad de Chile en 1943. Este grupo de tecnócratas, constituidos en una verdadera logia, acordaron secretamente antes de las elecciones de 1952, unificarse para dar cuerpo al nuevo servicio en torno a aquéllos cuyo candidato venciese en dichas elecciones. Así lo hicieron y ocuparon los puestos principales, protegidos por la autonomía política del SNS, cuyo Director General era nombrado por el Presidente de la República pero con acuerdo del Senado. Un Consejo Directivo con la presencia de la facultad de medicina de la Universidad del Estado, de los usuarios y de las entidades de la previsión social, debía supervisar y acordar las grandes líneas de política.

Múltiples tareas enfrentó este grupo de tecnócratas de alta formación, además de sobrevivir políticamente en un mar adverso. Por de pronto unificar a casi 30 mil funcionarios que provenían de casi 20 servicios dispersos, crear una política de salud integral, mejorar la calidad de los recursos humanos, mejorar la cobertura hacia lo rural y las zonas de pobreza, enfrentar los problemas prevalentes, infecciones y la salud materno-infantil.

No fue fácil esta puesta en marcha pues, además, la opinión pública, los políticos y naturalmente la clase médica, privilegiaban la atención médica curativa por encima de lo preventivo, a lo cual se buscaba dedicar con fuerza por parte de sus fundadores. Decía el doctor Abraham Horwitz, su primer Subdirector Técnico:

Es artificial la segregación de las funciones curativas y preventivas. La medicina es una y se realiza mediante la integración de funciones de reparación, protección y fomento de la salud. Su objeto no es ya el paciente fragmentado por la especialización; es el hombre en su integridad bio-sicológica y social, es la persona con sus sentimientos, costumbres, creencias, tensiones y temores, es el ser social por naturaleza, en recíproca acción y reacción con su medio. ${ }^{7}$

El SNS asumía la visión ecológica de la salud y, contra viento y marea, la defendía en sus primeros difíciles e incomprendidos pasos.
Entre las acciones que se realizaron durante la primera década de acción del SNS están:

- Extensión de la cobertura hacia zonas apartadas y rurales mediante una red de consultorios y hospitales comunales atendidos por médicos generales de zona.

- Relación docente asistencial con las facultades de medicina del Estado.

- Implementación progresiva de programas de salud especialmente en el área madre y niño.

Sin embargo, el impacto epidemiológico tardó en verse en las estadísticas de morbilidad y mortalidad, especialmente en las infantiles, cuya latencia fue más larga que la esperada por los fundadores de la nueva institucionalidad.

Como ustedes pueden ver, este proceso de construcción de un servicio o sistema de salud, incluyó los tres elementos esenciales que se necesitan para lograrlo:

- $\quad$ Mística, sentido de misión, compromiso.

- Ciencia, valoración objetiva de la realidad y ensayo de soluciones eficaces.

- Política, en el mejor y no tan buen sentido, capacidad de negociación y de generar acuerdos, el arte de los posibles.

\section{Las políticas de atención al niño}

Esta nueva estructura, si bien dotada de principios y tecnócratas astutos, era como un gran hardware en busca de softwares que le dieran motivos más precisos y específicos de acción, objetivos sanitarios y sociales. Por ello, un campo privilegiado donde se dio esta interacción y pauta de acción, fue en el de las políticas de atención de la salud infantil.

Como pediatra en lo clínico, les puedo contar también algunas historias vividas y algunas lecciones aprendidas.

Cuando la UNICEF lanzó su iniciativa GOBI a favor de la infancia en 1980, el Servicio Nacional de Salud chileno llevaba casi 30 años de trabajo en una estrategia basada en la atención materno-infantil. GOBI era la sigla para Growth Monitoring, es decir Crecimiento, Desarrollo y Nutrición, Oral Rehydration Therapy para la diarrea infantil y la deshidratación, en esa época la primera causa de mortalidad, Birth Spacing por espaciamiento de los nacimientos y lactancia materna como uno de los métodos para obtenerlo, y la I por Inmunizaciones, es decir el Programa Ampliado de Inmunizaciones. Un buen acrónimo para una estrategia 
de intervenciones fundamentales a favor de la sobre-

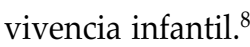

El SNS a su vez había nacido formalmente en 1952, pero como ocurre en el desarrollo de los sistemas de salud, era el tributario de varias iniciativas anteriores: Protección de la Infancia (Protinfa) de 1942, el Programa de Leche del Servicio de Seguro Social, de la Dirección de Salubridad y de varias otras estructuras y programas dispersos. En 1952, la tasa de mortalidad infantil (TMI) era de 132 por mil nacidos vivos, la pediatría científica estaba en una etapa de creciente desarrollo y sus principales maestros y grupos reconocían la importancia del medio ambiente físico, económico, social y cultural en la génesis de las enfermedades como en la necesidad de considerar integralmente esta multifactorialidad en la búsqueda de soluciones. $^{9}$

De esta forma, conscientes de que la pobreza y el medio ambiente insalubre eran las determinantes, desde la pediatría se lanzaron las primeras iniciativas de extensión de la cobertura hacia la comunidad, incluso antes del SNS a través de las unidades sanitarias, primer esbozo de centros de atención primaria en nuestro país en la década de los 40. En ellos, la puericultura, la atención de morbilidad pediátrica, el control de la desnutrición y las vacunas, junto con una progresiva atención de la función reproductiva de la madre, fueron el pilar del desarrollo de la incipiente extensión de cobertura.

El Centro de Adiestramiento e Investigación Materno Infantil (CAIMI), entidad mixta entre el SNS y la Cátedra de Pediatría del Hospital Arriarán, fue desde inicios de la década de los 50 el cerebro y cuerpo de diseño y experimentación en iniciativas de salud infantil que posteriormente se expandirían hacia el resto del país en la década de los $60 .{ }^{*}$ Los temas ahí estudiados e intervenidos en forma experimental en una población de la ciudad de Santiago reflejaban la patología prevalente de la época: desnutrición y alimentación del niño, diarrea aguda y deshidratación, enfermedades respiratorias y otras infecciones frecuentes, parasitosis, etcétera. En cuanto a los modelos de intervención estaban incorporados aspectos de manejo logístico y administrativo, una clara orientación hacia lo ambulatorio sin dejar de lado lo nosocomial. Los asuntos relativos a la investigación y la capacitación eran también centrales en las actividades de este

\footnotetext{
* Meneghello J. Experiencia y reflexiones sobre la enseñanza de Pediatría. Discurso de incorporación a la Academia Chilena de Medicina, 1986.
}

centro, siendo dirigidos tanto al personal profesional como al auxiliar.

Algunos de los éxitos reconocidos nacional e internacionalmente de este centro de investigación y adiestramiento fueron el desarrollo de la hidratación oral en forma ambulatoria, el control sistemático de la desnutrición con los primeros patrones y estándares del desarrollo normal, el planteamiento de subprogramas específicos para estos temas, (desnutrición, atención neonatal, escolar), con normas específicas de atención y orientadas a lo ambulatorio.

En suma, es posible decir que gran parte de la orientación del SNS en la década de 1960, aquella que daría cobertura a los problemas materno-infantiles, se caracterizó por la integración de la enseñanza, la investigación y la atención, todo en el entorno de lo comunitario y lo ambulatorio.

Las estrategias internacionales o promovidas por organismos internacionales, dan cuenta hoy, a inicios del siglo XXI de esta concepción que tuvieron los padres fundadores de la medicina social chilena hace más de 50 años atrás. Así, la OMS promueve el Manejo Integrado de Problemas de Salud de la Niñez por una parte, o propone utilizar la progresiva cobertura de las estructuras de vacunación, especialmente la relacionada con el esfuerzo para erradicar la poliomielitis, para ir incorporando otras acciones de beneficio en la salud infantil, como la entrega de vitamina A como protector contra las infecciones en la misma actividad. Todo ello con el propósito final de desarrollar estructuras de atención de salud que sean capaces de ir incorporando otros programas específicos para enfermedades o riesgos prevalentes en cada región. ${ }^{10}$

\section{Elementos y estrategias de éxito}

Después de la práctica, vienen la reflexión y la evaluación en esta historia ocurrida al interior de nuestro Sistema Nacional de Servicios de Salud (SNSS), caben algunas consideraciones.

Los componentes de la política que a mi juicio han dado a nuestro país una situación exitosa en la atención materno-infantil y que relacionan claramente el sistema de salud con la atención en esta área, son los siguientes: ${ }^{11}$

1. Una concepción integral de la salud, su relación con el medio y la necesidad de abordarla sistémicamente. Antes de iniciar las grandes modificaciones o construcciones de modelos de atención, y desde muy antiguo, los médicos han conocido la relación entre pobreza y morbilidad y mortalidad. Las leyes sanitarias de mediados del siglo XIX 
en Inglaterra fueron un ejemplo de ello, así como las concepciones de Virchow al ir más allá y decir que la búsqueda de la salud de los pobres era la forma más elevada de hacer política.

Desde la pediatría se ha observado, con especial énfasis y fuerza, la relación estrecha que existe entre los riesgos y daños a la salud con zonas y condiciones de pobreza. De esta forma, la mayor parte de los programas y modelos de intervención han sido desarrollados siempre privilegiando el trabajo con las comunidades pobres, ya sea en zonas urbanas o rurales.

2. Una visión integral de la atención de salud. Conforme con la concepción integral del fenómeno salud y enfermedad, un abordaje igualmente integrado se hacía una consecuencia lógica. Los conceptos de prevención, clásicos o modernos, han estado siempre presentes en las políticas de cuidado de la salud infantil. Partiendo por el conocimiento de la necesidad de proteger la vida desde la concepción, protegiendo el embarazo, preocupándose de la prevención del aborto y la multiparidad, la atención profesional del parto y el cuidado del recién nacido y así más adelante con los instrumentos específicos para cada riesgo a través del control sistemático del niño en su crecimiento, desarrollo y morbilidad. La captura de cada pareja madre-niño, a través del trabajo en comunidad, medios de comunicación, ambiente escolar y de incentivos como la donación de leche y otros alimentos, además de la atención gratuita, fueron parte de este desarrollo de un sistema de atención integral de salud del niño al cual la población chilena fue progresivamente respondiendo en forma masiva. Así se pudieron alcanzar las coberturas cercanas a $100 \%$ en la atención profesional del parto, del recién nacido, de las inmunizaciones y de gran parte de las acciones de salud.

Una acción atrayente trae a la otra en un mecanismo sumatorio progresivo que ha permitido ir tejiendo la red de atención de salud maternoinfantil hacia el modelo integrado. El consultorio periférico, base de la atención primaria, se inició en nuestro país como experiencia piloto en la década de 1930, en la red del Seguro Social, posteriormente en las unidades sanitarias de barrio y luego en la atención primaria del SNS. Pensemos que la formulación de la estrategia de atención primaria internacional y la meta de salud para todos en el año 2000 ocurre en Alma Atta en 1978.

La integración de la red asistencial no se ha producido exclusivamente de acuerdo con el proceso normal de desarrollo del niño, sino también de acuerdo con las patologías prevalentes para las cuales se han ido desarrollando en el tiempo programas específicos.

3. Un equipo de salud con delegación de funciones. Otra de las claves del éxito está en que en el equipo de salud materno-infantil ha ido ocurriendo a lo largo de los años una progresiva incorporación de otros profesionales y una creciente delegación de funciones. La enfermera pediátrica ha sido esencial en lo ambulatorio y en lo hospitalario; la matrona, central en la extensión de cobertura de la atención de la madre en su función reproductiva, y la nutricionista en lo concerniente a desnutrición y alimentación equilibrada. La auxiliar entrenada también fue incorporada como un recurso humano, tanto en lo urbano como en lo rural, especialmente en el control del niño sano y la solución de problemas menores. Este equipo médico profesional integrado es bastante único en Chile y sigue siendo un ejemplo.

4. Investigación y capacitación en la acción. En todas las instancias, ambulatorias y hospitalarias, de la atención de salud del niño, la actitud permanente de investigar, recolectar información para resumir experiencias observadas que permiten sacar conclusiones y planificar adecuadamente los recursos ha sido una constante que está en la cultura de nuestro sistema. Igualmente, la capacitación en la acción en torno a los problemas y estrategias de los temas más frecuentes. Es siempre motivo de satisfacción visitar lugares apartados y ver cómo casi a todo nivel se guardan estadísticas, se conocen los casos y se planifican acciones con base numérica y doctrinaria.

5. Evaluación continua de las acciones e instrumentos utilizados. Este es otro elemento clave en el resultado, la cultura de la evaluación y del compromiso con los proyectos y metas decididas después de las evaluaciones que retroalimentan la teoría y la acción. Incluso, hay decenas de ejemplos de cómo los instrumentos que se utilizan, aparentemente simples, son permanentemente evaluados y adaptados para su reutilización. Ayer fueron las estrategias para combatir desnutrición y diarrea infantil, hoy lo son las que se usan para las infecciones respiratorias agudas (IRA), la atención del recién nacido y la de los niños con problemas congénitos, entre otras.

En el año 2000, la tasa de mortalidad infantil en Chile es de 10 por mil nacidos vivos, el crecimiento relativo de los problemas perinatales y congénitos, además de las infecciones agudas respiratorias, da cuenta 
de una parte substancial de sus causas, y requiere nuevamente de mística, ciencia y política.

\section{Conclusiones}

Hemos visto cómo en nuestra experiencia, al igual que en muchas otras, las fuerzas que generan la mística, es decir el espíritu encarnado en seres humanos, en líderes y héroes civiles, con suficiente grado de elaboración científica y con un manejo adecuado de la política, van construyendo estructuras y programas de salud para el beneficio de las poblaciones.

Nada sin embargo es definitivo y todo está en un perpetuo cambio; existen periodos, llamados de reforma, que aparecen como más llamativos, pero que en verdad son cumbres de procesos lentos, tortuosos y complejos que van sumando fuerza y acciones para el objetivo común del mito colectivo del Bien Común.

Para mí, la mejor expresión literaria de una política y su proceso, es la que escribe Italo Calvino en la Jornada de un escrutador electoral, en 1956:

Amerigo había aprendido que los cambios en política se producen por caminos largos y complicados, y que no era cosa de esperárselos de un día al otro, por un giro de la fortuna. Para él, como para muchos otros, la experiencia había significado volverse un poco pesimistas. Por otra parte, estaba la consigna de que es necesario seguir haciendo lo que se pueda, día a día. En política, como en todas las cosas de la vida, y para quien no sea un necio, sólo cuentan dos principios: no hacerse demasiadas ilusiones y no dejar de creer que cualquier cosa que hagas puede servir. ${ }^{12}$
Así creo que es la realidad.

Construiremos nuestros sistemas de salud con nuestras creencias, nuestros mitos y nuestros héroes, nuestros conocimientos y nuestras habilidades.

Creyendo en nuestra información.

Apoyando a nuestros hermanos.

Inspirándonos en héroes como José Luis Bobadilla quien nos mira desde lo alto, pero que vive con nosotros diariamente.

\section{Referencias}

1. Campbell J.The heroe with a thousand faces. Princeton, $N$ ueva Jersey. University Press, 1973; Bollingen Series.

2.C ampbell J.Transformations of myth through time. Ed: Perennial Library, N ueva York: Harper \& Row Publishers, 1990.

3. 0 MS Europe. Health for All targets, Copenhague: O MS, 1988.

4. Boletín de los Servicios de Salud Fusionados. Chillán: casa ed, agosto de 1940. En: Illanes MA. Historia social de la salud pública chilena 1880/1973. Santiago de Chile: Edit. Colectivo de Atención Primaria, 1993.

5.Allende S. La realidad médico-social chilena. Santiago de Chile: Ministerio de Salubridad, Previsión y A sistencia Social, 1939.

6. Bo letín de laAsociación Médica de Chile, Marzo de 1947. En: Illanes MA, op cit

7. Horwitz A. Medicina en Chile. Revista del SN S, octubre de 1956:23. 8.UN IC EF. State of theW orld Children Report 1980. N uevaYork:UN IC EF, 1980

9. Rosselot-Vicuña J. Protección de la salud del niño y la familia. En: Meneghello J, ed. Pediatría. 3aedición. Santiago de Chile: Editorial Mediterráneo, 1985.

10. 0 rganización Mundial de la Salud. Manejo integrado de los problemas de salud del niño. Ginebra, 2000.

11. Jiménez J.Atención pediátrica y sistemas de salud en Chile. Rev Chil Pediatr 2000;71 (5):377-379.

12. Calvino I. La jornada de un escrutador. Madrid: Editorial Alianza Tres, 1988. 